\title{
Numerical Simulation and Analysis of Multi-Heat Source Stirling Engine Combustion Chamber
}

\author{
Guihai Jiao ${ }^{1}$, Qifen $\mathrm{Li}^{1}$, Lei Zhang ${ }^{1}$, Jingjing $\mathrm{Xu}^{1}$, Jialin Zhao ${ }^{1}$, Junpeng Mao ${ }^{1}$, \\ Xiaoyu Liang ${ }^{1}$, Zongqin Hou ${ }^{1}$, Yuntao Zhu ${ }^{1}$ \\ ${ }^{1}$ College of energy and Mechanical Engineering, Shanghai University of Electric Power, China
}

KEYWORD: Stirling engine, combustion chamber, simulation, ANSYS

ABSTRACT: This paper uses ICEM and the FLUENT CFD module of ANSYS software to do numerical simulation of Sterling engine combustion chamber 3D model, to reflect the the turbulent flow characteristics of engine combustion chamber in the Sterling velocity field distribution, further, according to the analysis results, optimize the present engine temperature section combustion chamber structure to improve the flue gas flow to improve the heat transfer performance.

\section{INTRODUCTION}

The Stirling engine is an external combustion (heating) closed type piston engine and fuel has advantages of low wide source, has the advantages of simple structure, high thermal efficiency, low pollution, low noise, convenient maintenance, and so on, can be application and many in the field as a clean efficient dynamic power, promote the use of Stirling engine for energy-saving emission combustion chamber is more and more come from the design of the engine combustion chamber. This method requires a lot of experimental experience and statistical results, not only need a lot of basic research time, but also the experimental equipment needs expensive cost. Reduction and environmental protection has important significance ${ }^{[1]}$.

To explore a suitable heating method giving to Stiring engine in both high and medium temperature Status, we can use the software ANSYS.

This paper uses ICEM and the FLUENT CFD module of ANSYS software to do numerical simulation of engine combustion chamber Sterling 3D simulation, to reflect the the turbulent flow characteristics of engine combustion chamber in the Sterling velocity field distribution, furtherly, according to the analysis results, optimize the present engine temperature section combustion chamber structure to improve the flue gas flow to improve the heat transfer performance.

\section{THE MATHEMATICAL MODEL}

The main purpose is to evaluate and optimize the velocity field in the design of the combustion chamber. In the actual working condition, all kinds of boundary conditions and the experimental environment are constantly changing. In numerical simulation with fluent software, we must consider the actual working conditions of the multi variability, complexity and simplify. In order to facilitate the study, this paper made the following several assumptions ${ }^{[2]}$ :

(1).fluid is a constant, incompressible fluid;

(2).the boundary conditions is non permeable and non slip surface;

(3).there is no exchange of heat and mass between the outside and the wall of the pipe wall;

(4).neglecting the thermal effect of the fluid in the process of the flow due to its viscous dissipation; 
(5).the flow in the pipe inlet is in the full development stage of the steady turbulent flow, and the flue gas has finished the combustion process;

(6).the ambient pressure is zero, and the working fluid is air.

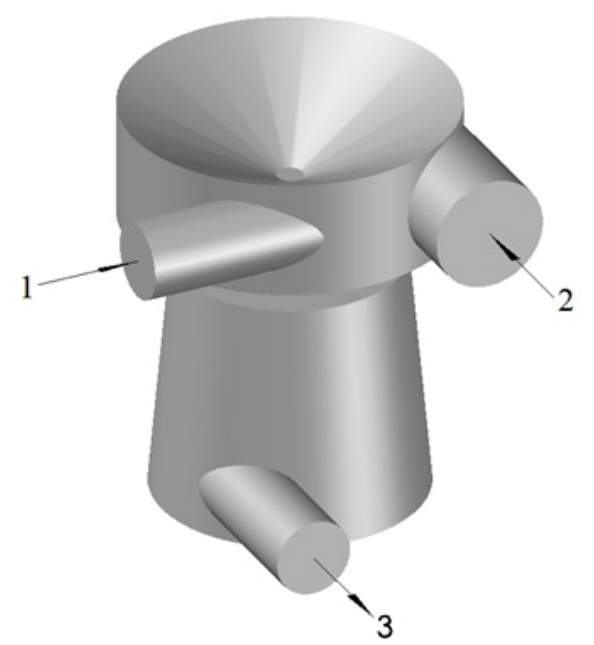

1: Waste heat (medium temperature gas) imports 2: high temperature gas imports $\quad 3:$ the export

Figure1. Three dimensional effect of multi heat source combustion chamber

$\mathrm{Re}$ is used to determine the flow state of the multi heat source combustion chamber, and the Re number formula is:

$$
\operatorname{Re}=\frac{u d}{v}
$$

$\operatorname{Re}<2000$ is in a laminar flow; $\operatorname{Re}>2000$ is in turbulent flow;

Waste heat gas imports: inlet temperature from $327^{\circ} \mathrm{C}$ the inlet velocity $\mathrm{u}=2 \mathrm{~m} / \mathrm{s}, \mathrm{d}=70 \times 10^{-3} \mathrm{~m}$;

Reference resources: $v=52.18 \times 10^{-6} \mathrm{~m} / \mathrm{s}$;

$\operatorname{Re}=\frac{2 \times 70 \times 10^{-5}}{52.1802 \times 10^{-8}}=2683.01>2000$

In the turbulent stage high temperature gas imports: import temperature of 927 degrees Celsius, import speed $\mathrm{u}=2 \mathrm{~m} / \mathrm{s}, \mathrm{d}=102 \times 10^{-3} \mathrm{~m}$; Refer resources: $v=161.04 \times 10^{-6} \mathrm{~m}^{2} / \mathrm{s}$;

$$
\operatorname{Re}=\frac{2 \times 102 \times 10^{-8}}{161.04 \times 10^{-6}}=1266.76<2000
$$

In the laminar stage. ${ }^{[3]}$

The two fluids are mixed in turbulence stage and laminar stage. because of the turbulence effects on the flow and heat transfer, so we use the k-e equation model; pressure based solution to guarantee the convergence stability; the pressure and velocity decoupling combined with semi implicit method for pressure linked equation algorithm; momentum, energy and turbulence parameters is solved us- 
ing the Second Order Upwind ${ }^{[4]}$; fluid inlet choose the velocity conditions; Waste heat (medium temperature gas) imports temperature setting to $600 \mathrm{k}$, the high temperature gas imports temperature setting to $1200 \mathrm{~K}$; the outlet using pressure boundary.

\section{ANALYSIS OF THE SIMULATION VELOCITY FIELD}

Two strands of air flow come into the mixing chamber, along with the combustion chamber cylinder body doing rotating flow from top to Bottom, finally through the exhaust of chamber flow into the external environment, in the flow of links, the airflow velocity changes as Figure2.

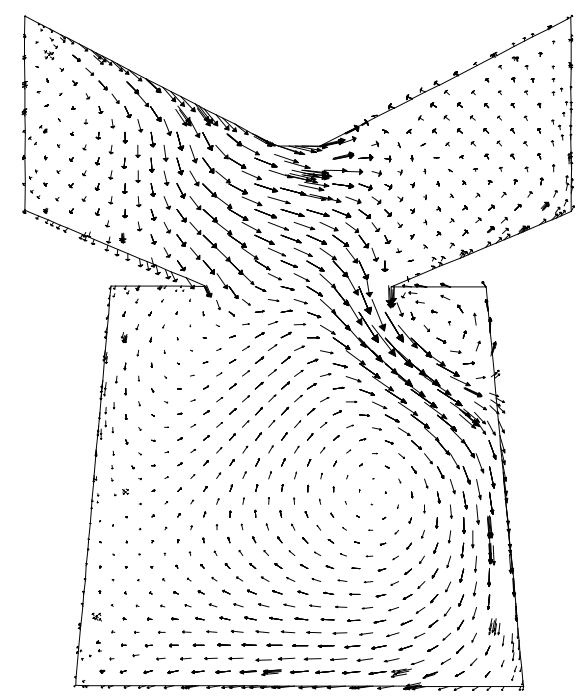

Figure $2 \mathrm{X} 1$ velocity vector diagram of vertical section

Airflow from the burning room is arranged on the upper part to the speed and the direction of the lower flow, speed in the narrow channel of the cylinder body, according to the Bernoulli principle, flow section narrowing, fluid pressure variable is small, the fluid flow velocity is increased, velocity vector arrows pointing here, maximum flow rate reached $7.5 \mathrm{~m} / \mathrm{s}$, combustion chamber of the narrow section design benefits is fluid into the heater of the heat exchange space, initial velocity was higher, and enhance the heat transfer capacity of pipe and the heating and high temperature gas flow fast flow and walk the heater temperature is more uniform.

\section{MULTI HEAT SOURCE COMBUSTION CHAMBER STRUCTURE OPTIMIZATION SCHEME}

On combustion chamber internal temperature field and the velocity field change analysis indicated that the combustion chamber to the heating pipe heating uniformity, but from the simulation results of multi heat source combustion chamber structure, there still has some room to improve, such as in the vertical section X1 velocity we can see that when the gas flow through the channel of the throat, in the edge of the rectangular flow produce concentrated vortex and the local temperature may different from others place. To solve the above questions, we can optimize the engine temperature section combustion chamber structure as shown in Figure 3.the optimization of multi heat source combustion chamber structure diagram, Figure 4 is multi heat source combustion chamber structural comparison between before and after optimization. 


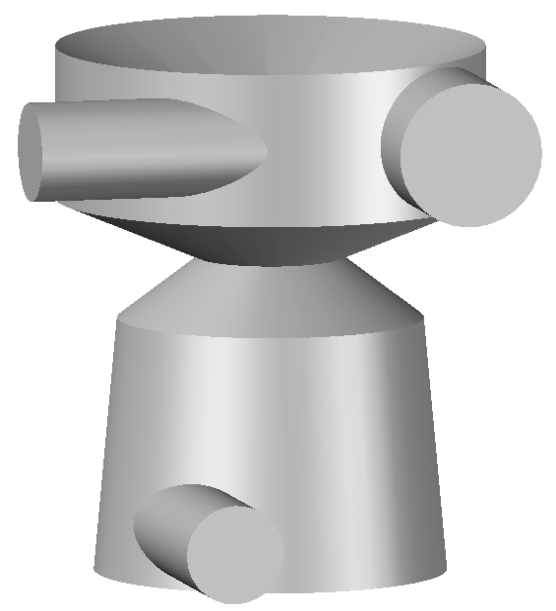

Figure 3 schematic diagram of optimum structure of multi heat source combustion chamber
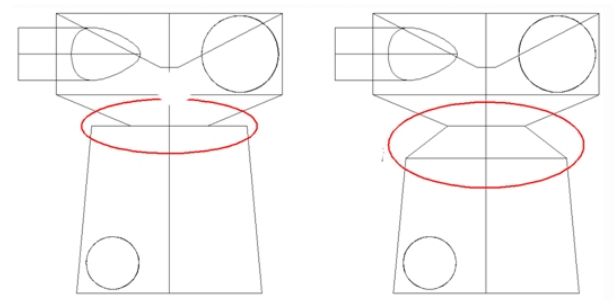

before optimization after optimization

Figure 4 multi heat source combustion chamber structural comparisons between before and after optimization

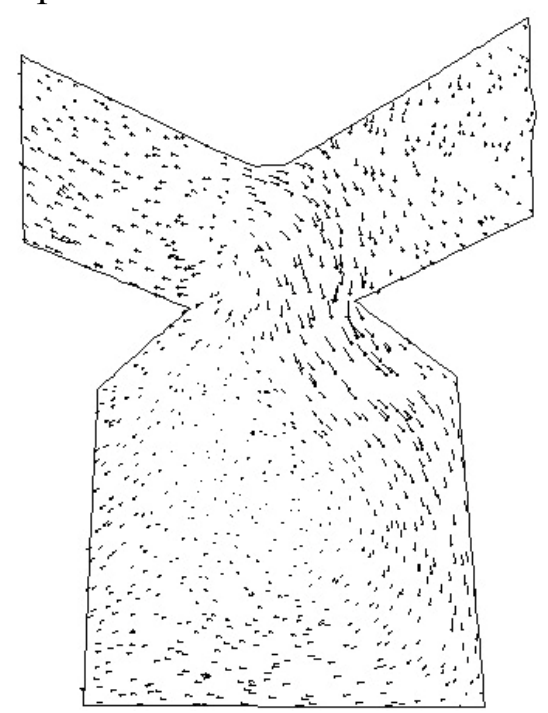

Fig. $5 \mathrm{X} 1$ velocity vector diagram of vertical section after optimization

After the optimization of multi heat source combustion chamber in the throat with a trapezoidal cross section to replace the original rectangular cross section. Figure 5 is after the optimization vertical section X1 velocity vector diagram. Contrast Figure 2, Figure 5 shows that because of the resection cavity at the top right edge, making the original vortex area destroyed, there is no obvious vortex, down flow area to expand the flow more smoothly. the angle between flow direction and the vertical direction decreases slightly.

So, this structure optimization has the following 2 advantages:

(1) The optimization of trapezoidal cross-section design can reduce the original rectangular cross section of the vortex, the kinetic energy loss of heat flow becomes smaller; reduce the local resis- 
tance loss, the hot air flow more smooth. The engine heater heat transfer effect are been strengthened.

(2) The heat flow to the trapezoid in rotation. then the trapezoidal lead heat flow to downward smoothly and effectively change the flow direction of the heat flow, let it point to the engine heater directly, also can improve the engine heater of heat transfer effect.

\section{CONCLUSION}

Author draw a 3D model of Sterling engine combustion chamber using the Auto CAD, and using ICEM model to mesh it, then import the meshed modeling to the software FLUENT. The conclusions of the velocity field about the fluid in combustion chamber are obtained:

(1) when air flows through the upper and lower chamber throat, flow section narrowing, so the fluid flow velocity increase, the highest velocity reaches $7.5 \mathrm{~m} / \mathrm{s}$, fluid into and heater heat exchange space, high speed, and enhance the heat transfer capacity of pipe and the heating, also in the high temperature gas rapid flow away the heater temperature is more uniform.

(2) According to simulation results of multi heat source of the combustion chamber and of multi heat source combustion chamber structure is optimized, the optimized combustion chamber in the throat with a trapezoidal cross section to replace the original rectangular cross section and to optimize the design of such that the flow of hot air more unobstructed, strengthening the engine heater and the heat transfer effect.

\section{REFERENCES}

[1] Haiwei Li \& Linsuo Shi \& Yaqi Li. 2010 (04).Development and application of Energy technology.

[2] Li Jia \& Zhaohong Fang \& Xinghua Qian. 2005. Higher heat transfer. Beijing: Higher Education Press.

[3] Wenquan Tao \& Shiming Yang. 2006. Heat transfer. Beijing: Higher Education Press.

[4] Wenquan Tao. 2009. Multi - scale numerical simulation of heat transfer and flow problems:

method and application. Beijing: Science Press. 